\title{
The study of "budget impact" of antiglaucoma medicines, recommended for inclusion in formulary and insurance lists
}

\author{
Makarenko O. V. ${ }^{1}$, Kryvoviaz O. V. ${ }^{2}$, Kryvoviaz S. O. ${ }^{3}$ \\ ${ }^{I}$ Department Of Social Medicine, Organization And Health Management, State Establishment "Dnipropetrovsk \\ Medical Academy Of Health Ministry Of Ukraine", Dnipro, Ukraine \\ ${ }^{2}$ Pharmacy department, National Pyrogov memorial medical university, Vinnytsia, Ukraine \\ ${ }^{3}$ Eye diseases department, National Pyrogov memorial medical university, Vinnytsia, Ukraine
}

\begin{abstract}
As a pathology that requires lifelong treatment, primary open-angle glaucoma (POAG) causes significant increase in direct (pharmacotherapy) and indirect costs (associated with partial or complete disability of patients with POAG). The work presents the results of pharmacoeconomic "budget impact" analysis of antiglaucoma medicines (AGMs), recommended for inclusion in the formulary (FL) and insurance (IL) lists of pharmaceutical servicing patients with POAG on the basis of results obtained from previous studies. The "budget impact" analysis is an important part of integrated pharmacoeconomic evaluation of the health care system with the results needed by public funding bodies, namely for compensation of costs for pharmacotherapy to patients and approval of documents for refunding. The results of pharmacoeconomic study of POAG treatment with medications, recommended for inclusion in FL and IL, allowed to indicate the POAG treatment regimens, which help not only save costs in context of perspective of budget impact, but also provide significant advantage in achievement of target intraocular pressure (IOP) (characterized by the lowest "cost-efficacy" rate); POAG pharmacotherapy regimens is considered cost-effective, which, requiring additional costs nevertheless provides greater efficacy in lowering IOP; dominant schemes, switching to which requires additional costs.
\end{abstract}

Keywords - primary open-angle glaucoma, antiglaucoma medicines, study of "budget impact", formulary and insurance lists.

\section{Introduction}

The expedience of overall pharmacoeconomic analysis of primary open-angle glaucoma (POAG) treatment is preconditioned by epidemiological indicators, which clearly indicate growing morbidity and disability caused by the disease, as well as its social, medical, and economic importance for both patients` and the national budget [1-4].

It`s well known, that POAG is characterized by abnormally high intraocular pressure (IOP), associated with hyperproduction or impaired aqueous humor outflow from the eye, which, in turn, leads to disturbance of visual function, and, if untreated, may lead to complete loss of vision. According to WHO, POAG is one of the primary causes of disability in sight. Prevalence and incidence of glaucoma is increasing every year in the world and in Ukraine. In addition to negative impact on patients` health, partial or complete loss of vision causes significant reduction in quality of life $[5,6]$.

As a pathology that requires lifelong treatment, POAG causes significant increase in direct (pharmacotherapy) and indirect costs (associated with partial or complete disability of patients with POAG). If AGMs are purchased at patients`expense, direct costs for POAG pharmacotherapy cause substantial costs to patients and their families. In that case, the choice of AGMs may be driven mostly by price rather than a real need for them, or the necessity to comply with doctor's prescriptions. Moreover, the pharmacy is not able to provide the whole range of medicines of group S01E "Antiglaucoma preparations and miotics." In this regard, the work presents the results of pharmacoeconomic "budget impact" analysis of AGMs, recommended for inclusion in the formulary (FL) and insurance (IL) lists of pharmaceutical servicing patients with POAG on the basis of results obtained from previous studies $[7,8]$.

Therefore, all above-mentioned facts testify the topicality of optimizing medical and pharmaceutical care for patients with POAG under results of pharmacoeconomic survey of (AGM) antiglaucoma regimens prescribed by ophthalmologists in Ukraine.

Apparently, the "budget impact" analysis is an important part of integrated pharmacoeconomic evaluation of the health care system with the results needed by public funding bodies, namely for compensation of costs for pharmacotherapy to patients and approval of documents for refunding. [9] 


\section{The Purpose of The Work}

The objective of the study was assessment of "budget impact" of alternative POAG treatment regimens, recommended for inclusion in the formulary (FL) and insurance (IL) lists, by "cost-effectiveness" method with due regard to the data obtained from previous studies.

\section{Materials And Study Methods}

Pharmacoeconomic analysis performed using the "budget impact" principle allows to assess influence of studied medical technique (pharmacotherapy methods) on the health care budget of Ukraine. The "budget impact" is assessed taking into account the economic effect (savings), spent on a particular treatment regimens. The "budget impact" analysis is a comparative one, since it is based on assessment of difference in economic benefits of evaluated treatment regimens, expressed in monetary terms.

The result of "budget impact" analysis has been calculated under formula 1:

$\mathrm{BIA}=\operatorname{Efec}(1)-\operatorname{Efec}(2)$,

where BIA - the result of "budget impact" analysis, UAH;

Efec (1) - overall economic effect of comparator treatment regimen, UAH;

Efec (2) - overall economic effect of alternative treatment regimen, UAH [10].

Taking into account different AGM regimens, we used for calculations the cost of DDD/1 patient/year, UAH, calculated on the basis the data obtained from previous studies [11].

For that purpose, using this technique allows us to calculate the savings or the need for additional costs in case of replacing one regimen with another.

Therefore, we have calculated indices for finding POAG treatment regimens, by switching to which patients can save money, and identifying those that require additional costs for patients against the comparator regimen.

\section{Results And Discussions}

"Budget impact" analysis considers only economic effect of using comparative treatment regimens, despite the therapeutic efficacy of certain AGMs, assessed in terms of lowering IOP (\%) [12].

It is, therefore, advisable to compare the "budget impact" analysis data with the results of pharmacoeconomic "cost - efficacy" study in order to make possible the comparative assessment of not solely economic benefits, but also pharmacotherapeutic efficacy of different POAG treatment regimens (Table 1).

Table 1: Results of the "budget impact" analysis, counting on "cost-efficacy" index

\begin{tabular}{|c|c|c|c|}
\hline $\begin{array}{l}\text { Composition of comparator } \\
\text { regimen }\end{array}$ & Composition of study regimen & $\begin{array}{l}\text { "Budget impact" } \\
\text { analysis (BIA), UAH }\end{array}$ & $\triangle \mathrm{CER}$ \\
\hline \multirow{5}{*}{$\begin{array}{l}\text { Arutimol eye drops } 0.5 \%, 5 \\
\text { ml, Chauvin ankerpharm } \\
\text { (Germany) }\end{array}$} & $\begin{array}{l}\text { Lanotan eye drops } 0.05 \mathrm{mg} / \mathrm{ml}, 2.5 \mathrm{ml} \text { No. 1, VAT } \\
\text { "Farmak" (Ukraine) }\end{array}$ & -1208.44 & -6.124988 \\
\hline & $\begin{array}{l}\text { Travatan eye drops, } 40 \mu \mathrm{g} / \mathrm{ml}, 2.5 \mathrm{ml} \text { in drop } \\
\text { dispenser No. } 1 \text {, Alcon-Couvreur (Belgium) }\end{array}$ & -1689.37 & -6.459630 \\
\hline & $\begin{array}{l}\text { Arutimol eye drops } 0.5 \%, 5 \mathrm{ml} \text {, Chauvin } \\
\text { ankerpharm (Germany) }+ \text { Taflotan eye drops } 15 \\
\mu \mathrm{g} / \mathrm{ml}, 2.5 \mathrm{ml} \text { No. } 1 \text {, Santen (Finland) }\end{array}$ & -1289.47 & -2.631650 \\
\hline & $\begin{array}{l}\text { Arutimol eye drops } 0.5 \%, 5 \mathrm{ml} \text {, Chauvin } \\
\text { ankerpharm (Germany) + Lanotan eye drops } 0.05 \\
\mathrm{mg} / \mathrm{m}, 12.5 \mathrm{ml} \text { No. 1, VAT "Farmak" (Ukraine) }\end{array}$ & -2357.17 & -4.689860 \\
\hline & $\begin{array}{l}\text { Azopt Eye drops, } 10 \mathrm{mg} / \mathrm{ml}, 5 \mathrm{ml} \text { in drop } \\
\text { dispenser DROP-TAINER@ No. 1, Alcon- } \\
\text { Couvreur (Belgium) + Taflotan eye drops } 15 \\
\mu \mathrm{g} / \mathrm{ml}, 2.5 \mathrm{ml} \text { No. } 1 \text {, Santen (Finland) }\end{array}$ & -2411.04 & -4.854491 \\
\hline \multirow{5}{*}{$\begin{array}{l}\text { Lanotan eye drops } 0.05 \\
\text { mg/ml, } 2.5 \mathrm{ml} \text { No. 1, VAT } \\
\text { "Farmak" (Ukraine) }\end{array}$} & $\begin{array}{l}\text { Arutimol eye drops } 0.5 \%, 5 \mathrm{ml}, \quad \text { Chauvin } \\
\text { ankerpharm (Germany) }\end{array}$ & 1208.44 & 6.124988 \\
\hline & $\begin{array}{l}\text { Travatan eye drops, } 40 \mu \mathrm{g} / \mathrm{ml}, 2.5 \mathrm{ml} \text { in drop } \\
\text { dispenser No. 1, Alcon-Couvreur (Belgium) }\end{array}$ & -480.93 & -0.33464 \\
\hline & $\begin{array}{l}\text { Arutimol eye drops } 0.5 \%, 5 \mathrm{ml} \text {, Chauvin } \\
\text { ankerpharm (Germany) }+ \text { Taflotan eye drops } 15 \\
\mu \mathrm{g} / \mathrm{ml}, 2.5 \mathrm{ml} \text { No. } 1 \text {, Santen (Finland) }\end{array}$ & -81.03 & 3.493338 \\
\hline & $\begin{array}{l}\text { Arutimol eye drops } 0.5 \%, 5 \mathrm{ml} \text {, Chauvin } \\
\text { ankerpharm (Germany) + Lanotan eye drops } 0.05 \\
\mathrm{mg} / \mathrm{ml}, 2.5 \mathrm{ml} \text { No. 1, VAT "Farmak" (Ukraine) }\end{array}$ & -542.25 & 2.869602 \\
\hline & $\begin{array}{l}\text { Azopt Eye drops, } 10 \mathrm{mg} / \mathrm{ml}, 5 \mathrm{ml} \text { in drop } \\
\text { dispenser DROP-TAINER }{ }^{\circ} \text { No. } 1, \text { Alcon- } \\
\text { Couvreur (Belgium) + Taflotan eye drops } 15 \\
\mu \mathrm{g} / \mathrm{ml}, 2.5 \mathrm{ml} \text {, No. } 1 \text { Santen (Finland) }\end{array}$ & -1202.60 & 1.270497 \\
\hline $\begin{array}{l}\text { Travatan eye drops, } 40 \\
\mu \mathrm{g} / \mathrm{ml}, \quad 2.5 \mathrm{ml} \text { in drop }\end{array}$ & $\begin{array}{l}\text { Arutimol eye drops } 0.5 \%, 5 \mathrm{ml}, \text { Chauvin } \\
\text { ankerpharm (Germany) }\end{array}$ & 1689.37 & 6.459625 \\
\hline
\end{tabular}




\begin{tabular}{|c|c|c|c|}
\hline \multirow[t]{4}{*}{$\begin{array}{llll}\text { dispenser } & \text { No. } & 1, & \text { Alcon- } \\
\text { Couvreur (Belgium) } & \end{array}$} & $\begin{array}{l}\text { Lanotan eye drops } 0.05 \text { mg/ml, } 2.5 \text { ml, No. } 1 \text { VAT } \\
\text { "Farmak" (Ukraine) }\end{array}$ & 480.93 & 0.334637 \\
\hline & $\begin{array}{l}\text { Arutimol eye drops } 0.5 \%, 5 \mathrm{ml} \text {, Chauvin } \\
\text { ankerpharm (Germany) }+ \text { Taflotan eye drops } 15 \\
\mu \mathrm{g} / \mathrm{ml}, 2.5 \mathrm{ml} \text { No. } 1 \text {, Santen (Finland) }\end{array}$ & 399.90 & 3.827975 \\
\hline & $\begin{array}{l}\text { Arutimol eye drops } 0.5 \%, 5 \mathrm{ml} \text {, Chauvin } \\
\text { ankerpharm (Germany) + Lanotan eye drops } 0.05 \\
\mathrm{mg} / \mathrm{ml}, 2.5 \mathrm{ml} \text { No. 1, VAT "Farmak" (Ukraine) }\end{array}$ & -61.32 & 3.204239 \\
\hline & $\begin{array}{l}\text { Azopt Eye drops, } 10 \mathrm{mg} / \mathrm{ml}, 5 \mathrm{ml} \text { in drop } \\
\text { dispenser DROP-TAINER } ® \text { No. 1, Alcon- } \\
\text { Couvreur (Belgium) + Taflotan eye drops } 15 \\
\mu \mathrm{g} / \mathrm{ml}, 2.5 \mathrm{ml} \text { No. } 1 \text {, Santen (Finland) }\end{array}$ & -721.67 & 1.605134 \\
\hline \multirow{5}{*}{$\begin{array}{l}\text { Arutimol eye drops } 0.5 \%, 5 \\
\mathrm{ml}, \text { Chauvin ankerpharm } \\
\text { (Germany) + Taflotan eye } \\
\text { drops } 15 \mu \mathrm{g} / \mathrm{ml}, 2.5 \mathrm{ml} \text { No. } \\
\text { 1, Santen (Finland) }\end{array}$} & $\begin{array}{l}\text { Arutimol eye drops } 0.5 \%, 55 \mathrm{ml}, \quad \text { Chauvin } \\
\text { ankerpharm (Germany) }\end{array}$ & 1289.47 & 2.631650 \\
\hline & $\begin{array}{l}\text { Lanotan eye drops } 0.05 \mathrm{mg} / \mathrm{ml}, 2.5 \mathrm{ml} \mathrm{No} \text {. 1, VAT } \\
\text { "Farmak" (Ukraine) }\end{array}$ & 81.03 & -3.493340 \\
\hline & $\begin{array}{l}\text { Travatan eye drops, } 40 \mu \mathrm{g} / \mathrm{ml}, 2.5 \mathrm{ml} \text { in drop } \\
\text { dispenser No. 1, Alcon-Couvreur (Belgium) }\end{array}$ & -399.90 & -3.827980 \\
\hline & $\begin{array}{l}\text { Arutimol eye drops } 0.5 \%, 5 \mathrm{ml} \text {, Chauvin } \\
\text { ankerpharm (Germany) + Lanotan eye drops } 0.05 \\
\mathrm{mg} / \mathrm{ml}, 2.5 \mathrm{ml} \text { No. 1, VAT "Farmak" (Ukraine) }\end{array}$ & -461.22 & -0.623736 \\
\hline & $\begin{array}{l}\text { Azopt Eye drops, } 10 \mathrm{mg} / \mathrm{ml}, 5 \mathrm{ml} \text { in drop } \\
\text { dispenser DROP-TAINER } ® \text { No. 1, Alcon- } \\
\text { Couvreur (Belgium) + Taflotan eye drops } 15 \\
\mu \mathrm{g} / \mathrm{ml}, 2.5 \mathrm{ml} \text { No. 1, Santen (Finland) }\end{array}$ & -1121.57 & -2.222841 \\
\hline \multirow{5}{*}{$\begin{array}{l}\text { Arutimol eye drops } 0.5 \%, 5 \\
\mathrm{ml} \text {, Chauvin ankerpharm } \\
\text { (Germany) + Lanotan eye } \\
\text { drops 0.05 } \mathrm{mg} / \mathrm{ml}, 2.5 \mathrm{ml} \\
\text { No. 1, VAT "Farmak" } \\
\text { (Ukraine) }\end{array}$} & $\begin{array}{l}\begin{array}{l}\text { Arutimol eye drops } \\
\text { ankerpharm (Germany) }\end{array} \\
\text { ank }\end{array}$ & -2357.17 & -4.689860 \\
\hline & $\begin{array}{l}\text { Lanotan eye drops } 0.05 \mathrm{mg} / \mathrm{ml}, 2.5 \mathrm{ml} \mathrm{No.} \mathrm{1,} \mathrm{VAT} \\
\text { "Farmak" (Ukraine) }\end{array}$ & 542.25 & -2.869600 \\
\hline & $\begin{array}{l}\text { Travatan eye drops, } 40 \mu \mathrm{g} / \mathrm{ml}, 2.5 \mathrm{ml} \text { in drop } \\
\text { dispenser No. 1, Alcon-Couvreur (Belgium) }\end{array}$ & 61.32 & -3.204240 \\
\hline & $\begin{array}{l}\text { Arutimol eye drops } 0.5 \%, 5 \mathrm{ml} \text {, Chauvin } \\
\text { ankerpharm (Germany) }+ \text { Taflotan eye drops } 15 \\
\mu \mathrm{g} / \mathrm{ml}, 2.5 \mathrm{ml} \text { No. 1, Santen (Finland) }\end{array}$ & 461.22 & 0.623736 \\
\hline & $\begin{array}{l}\text { Azopt Eye drops, } 10 \mathrm{mg} / \mathrm{ml}, 5 \mathrm{ml} \text { in drop } \\
\text { dispenser DROP-TAINER } ® \text { No. 1, Alcon- } \\
\text { Couvreur (Belgium) + Taflotan eye drops } 15 \\
\mu \mathrm{g} / \mathrm{ml}, 2.5 \mathrm{ml} \text { No. 1, Santen (Finland) }\end{array}$ & -660.35 & $-1,599105$. \\
\hline \multirow{5}{*}{$\begin{array}{l}\text { Azopt eye drops, } 10 \mathrm{mg} / \mathrm{ml} \text {, } \\
5 \mathrm{ml} \text { in drop dispenser } \\
\text { DROP-TAINER } ® \text { No. } 1 \text {, } \\
\text { Alcon-Couvreur (Belgium) + } \\
\text { Taflotan eye drops } 15 \mu \mathrm{g} / \mathrm{ml} \text {, } \\
2.5 \mathrm{ml} \text { No. } 1, \text { Santen } \\
\text { (Finland) }\end{array}$} & $\begin{array}{l}\begin{array}{l}\text { Arutimol eye drops } \\
\text { ankerpharm (Germany) }\end{array} \\
\text { ank }\end{array}$ & 2411.04 & 4.854491 \\
\hline & $\begin{array}{l}\text { Lanotan eye drops } 0.05 \mathrm{mg} / \mathrm{ml}, 2.5 \mathrm{ml} \text { No. 1, VAT } \\
\text { "Farmak" (Ukraine) }\end{array}$ & 1202.60 & -1.270500 \\
\hline & $\begin{array}{l}\text { Travatan eye drops, } 40 \mu \mathrm{g} / \mathrm{ml}, 2.5 \mathrm{ml} \text { in drop } \\
\text { dispenser No. 1, Alcon-Couvreur (Belgium) }\end{array}$ & 721.67 & -1.605130 \\
\hline & $\begin{array}{l}\text { Arutimol eye drops } 0.5 \%, 5 \mathrm{ml} \text {, Chauvin } \\
\text { ankerpharm (Germany) }+ \text { Taflotan eye drops } 15 \\
\mu \mathrm{g} / \mathrm{ml}, 2.5 \mathrm{ml} \text { No. 1, Santen (Finland) }\end{array}$ & 1121.57 & 2.222841 \\
\hline & $\begin{array}{l}\text { Arutimol eye drops } 0.5 \%, 5 \mathrm{ml}, \text { Chauvin } \\
\text { ankerpharm (Germany) + Lanotan eye drops } 0.05 \\
\mathrm{mg} / \mathrm{ml}, 2.5 \mathrm{ml} \text { No. } 1 \text { VAT, "Farmak" (Ukraine) }\end{array}$ & 660.35 & 1.599105 \\
\hline
\end{tabular}

The results of pharmacoeconomic study of POAG treatment with medications, recommended for inclusion in FL and IL, indicate the following POAG treatment regimens, which help not only save costs in context of perspective of budget impact, but also provide significant advantage in achievement of target IOP (characterized by the lowest "cost-efficacy" rate):

- Arutimol eye drops 0.5\%, $5 \mathrm{ml}$, Chauvin ankerpharm (Germany) compared to Lanotan eye drops $0.05 \mathrm{mg} / \mathrm{ml}, 2.5 \mathrm{ml}$ No. 1, VAT "Farmak" (Ukraine);

- $\quad$ Arutimol eye drops $0.5 \%, 5 \mathrm{ml}$, Chauvin ankerpharm (Germany) compared to Travatan eye drops, $40 \mu \mathrm{g} / \mathrm{ml}$ , $2.5 \mathrm{ml}$ in drop dispenser No. 1 Alcon-Couvreur (Belgium);

- Lanotan eye drops $0.05 \mathrm{mg} / \mathrm{ml}, 2.5 \mathrm{ml}$ No. 1, VAT "Farmak" (Ukraine) compared to Travatan eye drops, $40 \mu \mathrm{g} / \mathrm{ml}, 2.5 \mathrm{ml}$ in drop dispenser No. 1, Alcon-Couvreur (Belgium);

- $\quad$ Arutimol eye drops $0.5 \%, 5 \mathrm{ml}$, Chauvin ankerpharm (Germany) + Taflotan eye drops $15 \mu \mathrm{g} / \mathrm{ml}, 2.5 \mathrm{ml}$ No. 1, Santen (Finland) compared to Travatan eye drops, $40 \mu \mathrm{g} / \mathrm{ml}, 2.5 \mathrm{ml}$ in drop dispenser No. 1, AlconCouvreur (Belgium);

- $\quad$ Arutimol eye drops $0.5 \%, 5 \mathrm{ml}$, Chauvin ankerpharm (Germany) + Taflotan eye drops $15 \mu \mathrm{g} / \mathrm{ml}, 2.5 \mathrm{ml}$ No. 1, Santen (Finland) compared to Arutimol eye drops $0.5 \%, 5 \mathrm{ml}$, Chauvin ankerpharm (Germany) + Lanotan eye drops 0.05 mg/ml, 2.5 ml No. 1 VAT, "Farmak" (Ukraine). 
Switching from below-mentioned POAG pharmacotherapy regimens is considered cost-effective, which, requiring additional costs nevertheless provides greater efficacy in lowering IOP:

- from Arutimol eye drops $0.5 \%, 5 \mathrm{ml}$, Chauvin ankerpharm (Germany) to Arutimol eye drops $0.5 \%, 5 \mathrm{ml}$, Chauvin ankerpharm (Germany) + Taflotan eye drops $15 \mu \mathrm{g} / \mathrm{ml}, 2.5 \mathrm{ml}$ No. 1, Santen (Finland) or Arutimol eye drops $0.5 \%, 5 \mathrm{ml}$, Chauvin ankerpharm (Germany) + Lanotan eye drops $0.05 \mathrm{mg} / \mathrm{ml}, 2.5 \mathrm{ml}$ No. 1 , VAT "Farmak" (Ukraine) or Azopt eye drops, $10 \mathrm{mg} / \mathrm{ml}, 5 \mathrm{ml}$ in drop dispenser DROP-TAINER® No. 1Alcon-Couvreur (Belgium) + Taflotan eye drops $15 \mu \mathrm{g} / \mathrm{ml}, 2.5 \mathrm{ml}$ No. 1, Santen (Finland);

- from Arutimol eye drops $0.5 \%, 5 \mathrm{ml}$ Chauvin ankerpharm (Germany) to Arutimol eye drops $0.5 \%, 5 \mathrm{ml}$, Chauvin ankerpharm (Germany) + Taflotan eye drops $15 \mu \mathrm{g} / \mathrm{ml}, 2.5 \mathrm{ml}$ No. 1, Santen (Finland);

- from Lanotan eye drops $0.05 \mathrm{mg} / \mathrm{ml}, 2.5 \mathrm{ml}$ No. 1, VAT "Farmak" (Ukraine) to Travatan eye drops, $40 \mu \mathrm{g} / \mathrm{ml}, 2.5 \mathrm{ml}$ in drop dispenser No. 1, Alcon-Couvreur (Belgium);

- from Arutimol eye drops $0.5 \%, 5 \mathrm{ml}$, Chauvin ankerpharm (Germany) + Taflotan eye drops $15 \mu \mathrm{g} / \mathrm{ml}, 2.5$ $\mathrm{ml}$ No. 1, Santen (Finland) or from Arutimol eye drops $0.5 \%, 5 \mathrm{ml}$, Chauvin ankerpharm (Germany) + Lanotan eye drops $0.05 \mathrm{mg} / \mathrm{ml}, 2.5 \mathrm{ml} \mathrm{No}$. 1, VAT "Farmak" (Ukraine) to Azopt eye drops, $10 \mathrm{mg} / \mathrm{ml}, 5$ $\mathrm{ml}$ in drop dispenser DROP-TAINER® No. 1, Alcon-Couvreur (Belgium) + Taflotan eye drops $15 \mu \mathrm{g} / \mathrm{ml}$, $2.5 \mathrm{ml}$ No. 1, Santen (Finland).

\section{Dominant schemes, switching to which requires additional costs are:}

- Arutimol eye drops 0.5\%, $5 \mathrm{ml}$, Chauvin ankerpharm (Germany) + Taflotan eye drops $15 \mu \mathrm{g} / \mathrm{ml}, 2.5 \mathrm{ml}$ No. 1, Santen (Finland) or Arutimol eye drops $0.5 \%, 5 \mathrm{ml}$, Chauvin ankerpharm (Germany) + Lanotan eye drops $0.05 \mathrm{mg} / \mathrm{ml}, 2.5 \mathrm{ml}$ No. 1, VAT "Farmak" (Ukraine) or Azopt eye drops, $10 \mathrm{mg} / \mathrm{ml}, 5 \mathrm{ml}$ in drop dispenser DROP-TAINER ${ }^{\circledR}$ No. 1, Alcon-Couvreur (Belgium) + Taflotan eye drops $15 \mu \mathrm{g} / \mathrm{ml}, 2.5 \mathrm{ml}$ No. 1, Santen (Finland) compared to Lanotan eye drops $0.05 \mathrm{mg} / \mathrm{ml}, 2.5 \mathrm{ml}$ No. 1 VAT, "Farmak" (Ukraine);

- Arutimol eye drops $0.5 \%, 5 \mathrm{ml}$, Chauvin ankerpharm (Germany) + Lanotan eye drops $0.05 \mathrm{mg} / \mathrm{ml}, 2.5 \mathrm{ml}$ No. 1, VAT "Farmak" (Ukraine) or Azopt eye drops, $10 \mathrm{mg} / \mathrm{ml}, 5 \mathrm{ml}$ in drop dispenser DROP-TAINER® No. 1, Alcon-Couvreur (Belgium) + Taflotan eye drops $15 \mu \mathrm{g} / \mathrm{ml}, 2.5 \mathrm{ml} \mathrm{No.} \mathrm{1,} \mathrm{Santen} \mathrm{(Finland)} \mathrm{compared}$ to Travatan eye drops, $40 \mu \mathrm{g} / \mathrm{ml}, 2.5 \mathrm{ml}$ in drop dispenser No. 1, Alcon-Couvreur (Belgium).

\section{Conclusion}

Therefore, using "budget impact" analysis makes possible to evaluate influence of changes in POAG treatment regimens on the cost of the disease therapy. Summarized information may be needed by professionals of health budget planning, insurance experts, heads of health institutions, and employers, which are required to make payments in temporary disability fund.

\section{References}

[1] Global Initiative for the Elimination of Avoidable Blindness: action plan 2006-2011, Available at: http://www.who.int /blindness/Vision2020_report.pdf

[2] S. V. Zbitneva, Zahvoruvanist' naselennia Ukrainy na hvoroby oka ta iiogo prydatkovoho aparatu, Visnyk social'noii hihieny ta organizacii ohorony zdoroviia Ukraiiny, 3, 2010, 14-18.

[3] O. V. Kryvoviaz, Farmakoterapiia hlaukomy: suchasnyi stan problemy, Farmakolohiia ta likarska toksykolohiia, 4-5 (35), 2013, 313.

[4] Primary open-angle glaucoma. Adapted clinical guidelines based on evidence (2011). Available at: http://www.dec.gov.ua/mtd/dodatki/816/816dod4_2.doc.

[5] O. Kryvoviaz, Antiglaucoma pharmacotherapy: analysis of treatment regimens and efficacy indicators, Science Rise, 3/4 (20), 2016, 46-50.

[6] O. V. Kryvoviaz, The quality of life of patients as a criterion of efficacy of primary open angle glaucoma therapy, IOSR Journal of Pharmacy and Biological Sciences (IOSR-JPBS), 11 (5), 2016, 12-14.

[7] O. Makarenko, O. Kryvoviaz, S. Kryvoviaz, Formulary and Insurance Lists of Medicines as a Means of Control of Quality of Medical Care to Patients with Primary Open-Angle Glaucoma, Quest Journals Journal of Research in Pharmaceutical Science, 5 (2), 2017, 01-04.

[8] A. V. Stepanenko, V. Ye. Blikhar, Intehratsiia formuliarnoi systemy likarskykh zasobiv u systemu standartiv u sferi okhorony zdorovia, Visnyk problem biolohii i medytsyny, 3 (1), 2014, 330-333.

[9] S. D. Sullivan, J. A. Mauskopf, F. Augustovski et al., Principles of good practice for budget impact analysis II: Report of the ISPOR Task Force on Good Research Practices - Budget Impact Analysis, Value Health, 17, 2014, 5-14.

[10] P. A. Vorobiev, M. V. Avksentieva, A. S. Iuriev et al., Kliniko-ekonomicheskii analiz (Moscow, New-diamed, 2004).

[11] O. V. Makarenko, O. V. Kryvoviaz, S. O. Kryvoviaz, Porivniannia kursovyh doz protyhlaukomnyh preparative u DDD /na rik / na 1 hvoroho ta iih vartosti dlia vyboru efektyvnoho ta ekonomichno dostupnoho likuvannia, Clinichna farmaciia, 3, 2014, 25-30.

[12] O. V. Kryvoviaz, Farmakoekonomichna ocinka likuvannia hlaukomy za metodom "vytraty-efektyvnist"”, Farmacevtychnii zhurnal, 2, 2016, 16-20. 